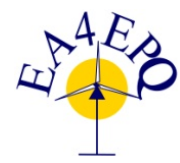

International Conference on Renewable Energies and Power Quality (ICREPQ'18)

Salamanca (Spain), $21^{\text {th }}$ to $23^{\text {th }}$ March, 2018

Renewable Energy and Pomer Quality. Fournal (RE\&PQJ)

ISSN 2172-038 X, No.16 April 2018

\title{
Electronic differential system for Light Electric Vehicles with two in- wheel motors
}

\author{
Clavero-Ordóñez, Lucía ${ }^{1}$, Fernández-Ramos, José ${ }^{1}$ and Gago-Calderón, Alfonso ${ }^{2}$ \\ ${ }^{1}$ Departmento de Electrónica \\ Escuela de Ingenierías Industriales, Universidad de Málaga \\ Complejo Tecnológico de Teatinos 2.2.41, 29071 Málaga (Spain) \\ Phone number:+0034 952 131441, e-mail: josefer@ctima.uma.es, \\ ${ }^{2}$ Departmento de Expresión Gráfica, Diseño y Proyectos \\ Escuela de Ingenierías Industriales, Universidad de Málaga \\ Calle Doctor Ortiz Ramos s/n, 29071 Málaga (Spain) \\ Phone number:+0034 951 952274, e-mail: agago@uma.es,
}

\begin{abstract}
.
This paper deals with the design, implementation and evaluation of an electronic differential system intended for light electric vehicles. Its operation is based on splitting the torque equally for two independent brushless DC motors installed in the same axis of the vehicle and directly coupled to the wheels. This configuration allows the motors to rotate at different speeds when the vehicle traces a curve. The system also detects and corrects the slipping of any traction wheel.

The main feature of the proposed system is that it does not require specific sensors to measure the steering angle and the speed of the drive wheels. Another important feature is that it is implemented using standard electric bicycle controllers and a general purpose Arduino platform. These components are very inexpensive and are available almost anywhere in the world.
\end{abstract}

\section{Key words}

Electric differential, Electronic Differential, Light Electric Vehicle, Mobility efficiency, Steering sensorless control

\section{Introduction}

Light Electric Vehicles (LEVs) are currently one of the most important alternatives to get the objective of sustainable urban mobility [1]. Although the concept of LEV is not perfectly defined, we can consider that it can be applied to those vehicles whose weight is of the same order as the total weight of the passengers for which they are designed. (Eg. $75 \mathrm{~kg}$ for one passenger vehicles and $150 \mathrm{~kg}$ for two passenger vehicles).

So, its main characteristic is the low weight, which results in a very low energy consumption and, therefore, a mobility efficiency superior to that conventional electric vehicles [2] - [3].

The LEVs most used today are two-wheeled models: electric bicycles, mopeds and motorcycles [4].
In addition to their reduced weight, another advantage of LEVs is that they have a single drive wheel. So, the control system and the motor (normally a hub motor) have low complexity and cost.

However, these vehicles have three major drawbacks: a high aerodynamic coefficient (that has a negative impact on their energy efficiency), the driver's exposure to the weather and low stability at low speeds.

The three-wheeled and closed body LEVs appear as a superior alternative to two-wheeled vehicles because they overcome these drawbacks [5]. They also have a single drive wheel, so both the control system and the motor are as simple and inexpensive as those of two-wheeled vehicles.

However, three-wheeled LEVs also have drawbacks. One of them is the low cornering stability, that limits the maximum speed under these conditions [6].

Although there are sophisticated solutions to improve the stability of tricycles such as tilt-wheeled designs [7], the most accepted solution to improve stability is the use of four-wheeled vehicles.

The power train of four-wheel vehicles is more complex than the power train of tricycles since it must allow that the two drive wheels rotate at different speeds when cornering. The mechanical system that allows this mode of operation is known as differential.

The use of mechanical differential is widespread in most conventional VEs and even in some LEVs [8], however, it has the drawback of being very heavy.

The mechanical differential is not suitable to be used in the LEVS due to its high weight. For these vehicles it is advantageous to implement an electrical or electronic differential (ED) system.

The main characteristics of the ED are [9]:

- There is no mechanical link between the two drive wheels. Each of them is coupled to an electric motor that is independently controlled. 
- The traction power is separately applied to each wheel by its controller.

- When cornering, the controller will apply less power to the inner wheel.

- The ED simulates a differential lock while the front wheels are driving straight paths.

The use of an ED also allows to improve the stability of tricycles with two drive wheels [10].

There are different ways to implement an ED. Some are complex, such as the "side slip control", which lies in the torque in each motor is regulated to improve the yaw rate of the vehicle [11]. However, the simplest way to implement an ED is to apply the same torque to each of the driving wheels [12].

Even in this simplest form of implementation, a controller of these characteristics presents a level of complexity quite superior to the standard controllers of electric bicycles, which are designed to regulate the power of a single motor. Therefore, the use of this type of low cost and widespread controllers should be discarded, with the consequent increase in the overall cost of the system.

Most of ED controllers require at least sensors to measure the speed of the driving wheels, the current of each motor and the steering angle [13].

However, some controller designs eliminate some of these sensors to gain simplicity at the expense of reducing some of their features. In this line, a controller without wheel speed sensors is proposed in [14] and another controller without steering angle sensor nor speed sensors is proposed in [15]. Both designs are applied to VEs driven with induction motors.

In [15] standard industrial frequency converters are used instead of the design of a new type of controller. This allows obtaining the advantages of higher reliability, low price and broad range of products and suppliers.

In this paper a design similar to the previous one is proposed, but applied to a LEV equipped with BLDC motors and standard electric bicycle controllers. The control system has been completed with the well-known Arduino platform, which allows the addition of special functions such as traction control and anti-lock wheel system.

\section{Work Methodology}

\section{A. Theory fundamentals.}

The principle of operation of the proposed ED is to ensure that the two motors of the power train deliver the same torque and can rotate at different speeds. In Figure 1a the torque-speed curves of a BLDC motor for different values of the applied voltage (equivalent to the duty cycle of the control signal) are shown. The operating point of the two motors when the vehicle is travelling in a straight line at medium speed is also represented (we assume that the two engines have identical characteristics and the torque set point $T_{C}$ is set by the accelerator).

When the steering system forces the vehicle to trace a curve, the torque of each motor $\left(T_{L}\right.$ and $\left.T_{R}\right)$ will be modified to allow each wheel to turn at a different speed (before the response of the control system occurs) (Figure 1b). Given this variation of the torque delivered to each wheel, the control system acts to reduce the duty cycle of the control signal of the inner wheel motor (left) and increase that of the outer wheel until the torque delivered by both motors returns to be equal to the set point set by the accelerator (figure 1c). In this new state, the speed of the motors is adequate to trace the curve correctly.

\section{B. Hardware}

Two versions of hardware have been developed to implement the ED proposed in this paper.

In the first version, only two sensors were used to measure the current applied to each motor. The torque delivered by a BLDC motor is proportional to its current, so it can be estimated by measuring that current. In this version there is no speed sensor available to measure the speed of the driven wheels. This has the disadvantage that abnormal situations, such as blocking or slipping of a wheel, can not be detected.

To overcome this problem a second hardware version has been made. In this version the signals of the Hall effect sensors of the BLDC motors have been used to estimate the rotation speed of the wheels. In this way, it has not been necessary to add any new speed sensor to the system.

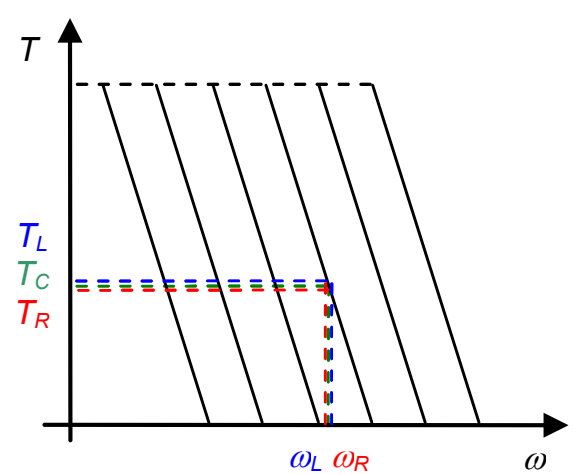

(a)

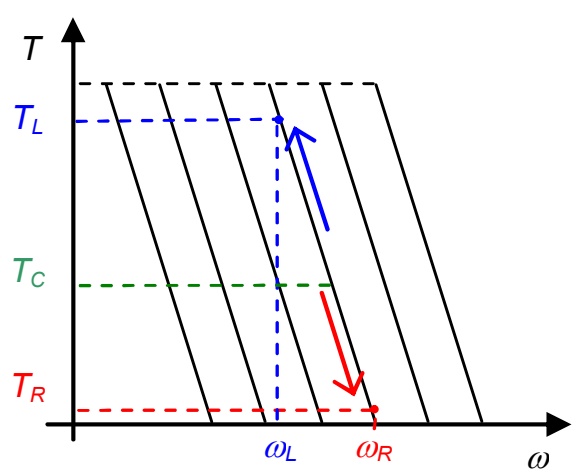

(b)

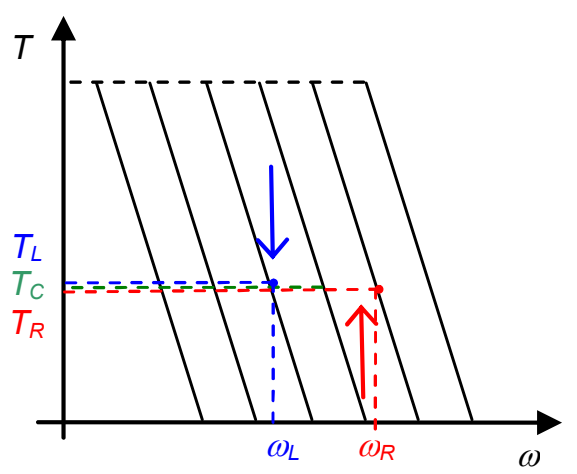

(c)

Figure 1. Example of operation of an ED with torque equalization in brushless DC motors ( $T_{C}$ : Torque setpoint; $T_{L}, \omega_{L}$ : Torque and speed of left motor; $T_{R}, \omega_{R}$ : Torque and speed of right motor) 


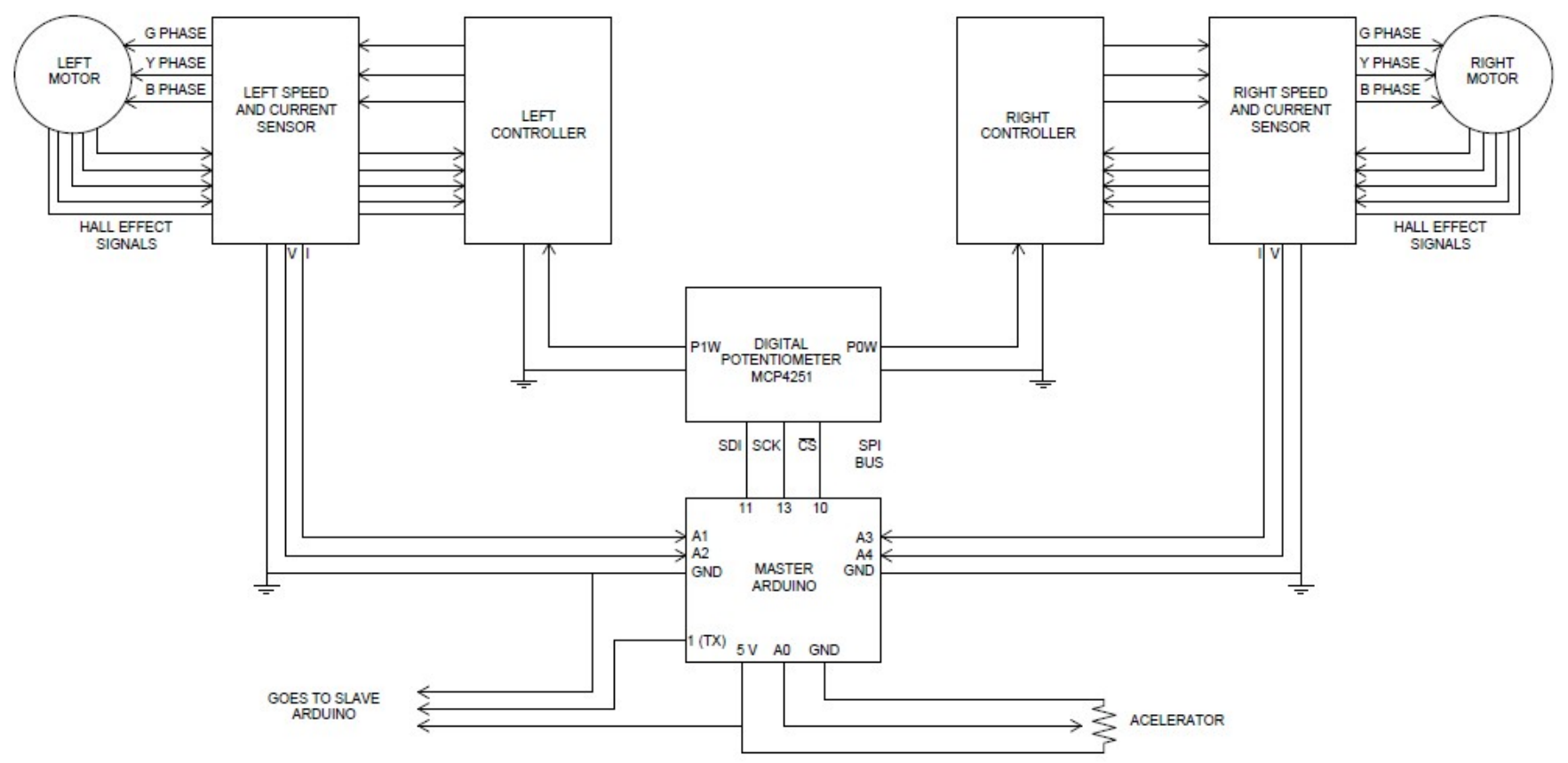

Figure 2. Hardware. General block diagram

Figure 2 shows the block diagram of the second version of the hardware. The main components of the hardware are the following:

\section{1) BLDC motors}

Two BLDC motors have been used for implementing the ED. The type of motor was the Nine Continent RH205B.

2) BLDC controllers.

Two Infineon 17A standard bicycle controllers were used.

3) Speed and current sensors

Speed and current sensors have been joined in a unique device for each wheel. We have used unidirectional "shunt" sensors to measure the current, while speed sensors utilize the Hall effect sensors which are already integrated in BLDC motors.

\section{4) Arduino platform}

The microcontroller used to implement the control algorithm is the Arduino UNO platform.

\section{5) Digital potentiometer}

Electric bicycle standard controllers use a potentiometer to set the duty cycle of the output transistors control signal. In this way the power delivered to the motor is regulated. This potentiometer establishes a voltage at the input of the controller that varies from $1 \mathrm{~V}$ to approximately $4 \mathrm{~V}$.

The potentiometer can be eliminated by applying directly to the input of the controller the output of a digital to analog converter (DAC). The Arduino Zero and Arduino DUE platforms have this type of outputs, so they could be used by connecting them directly to the controller input. This allows the automatic regulation of the voltage at the input of the controller at a level established by the control algorithm executed by the Arduino platform.

However, in our Hardware version we have used an Arduino UNO platform, which does not have DAC outputs. Therefore, the solution implemented has been the replacement of the potentiometers of the controller by digital potentiometers regulated by Arduino. We have used a chip MCP4251, which includes two digital potentiometers, one for each motor.

\section{Software}

\section{1) Main program}

The operation process is the following:

The accelerator sets the torque set point that both motors must deliver. This is received by the Arduino, which also reads the feedback of the current flowing through the motors, which is measured by the current sensors. Attending to the set point and the feedback, the program executes a Proportional-Integral control to obtain the optimal values of the control signals. These values are send to the digital potentiometers that regulates the output level of the motors controllers.

\section{2) Traction control}

Based on the minimum turning radius $\left(r_{\min }\right)$ that can be traced by the vehicle (the radius of the circumference that traces the outer wheel), the ratio of the speeds of the drive wheels must be within the range shown in the following equation:

$$
\frac{r_{\min }-d}{r_{\min }} \leq \frac{v_{\text {wheel } R}}{v_{\text {wheelL }}} \leq \frac{r_{\min }}{r_{\min }-d}
$$

Where $d$ is the distance between the drive wheels

The slip of any of the drive wheels is detected when the ratio of the speeds of both wheels is outside the range shown in equation (1). In this case, the traction control algorithm reduces the set point of the wheel that rotates at a higher speed until the ratio of the speeds of both wheels is again within that range.

This algorithm works well whenever only one of the drive wheels slips, but it is not useful when the two wheels do. 
This limitation can be overcome if the vehicle has a speed sensor coupled to any of the non-driven wheels, usually the front steering wheels.

Assuming a tricycle with a single front non-drive wheel and that this wheel does not slip, the equation that gives the limits of the ratio between the speed of any of the traction wheels and the front wheel are the following:

$$
\frac{2 \cdot r_{\min }-2 \cdot d}{2 \cdot r_{\min }-d} \leq \frac{v_{R}}{v_{F}} \leq \frac{2 \cdot r_{\min }}{2 \cdot r_{\min }-d}
$$

Where $v_{F}$ is the speed of the front wheel and $v_{R}$ the speed of any of the traction wheels.

The slip of any of the drive wheels is detected when the value of this ratio is outside those limits.

\section{System testing and results}

The system has been installed on a prototype (Figure 3) and then tested in order to check its performance. The prototype consists on a three-wheel vehicle derived of a bicycle with a bar support which holds the two rear wheels. BLDC motors are directly coupled to these wheels.

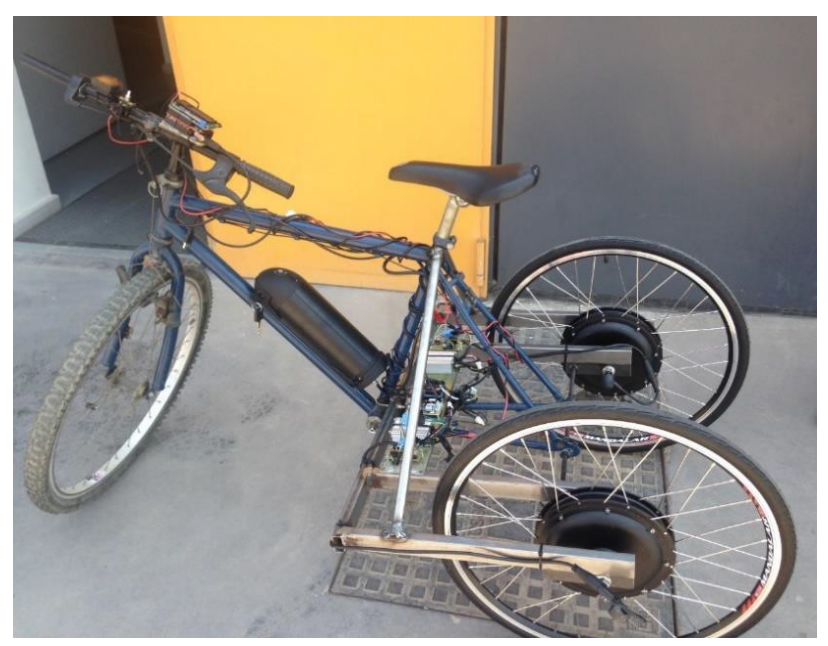

Figure 3

To check the performance of the ED, the vehicle has been subjected to the worst case test: a circular path with the minimum radius.

The vehicle has been driven on a circumference with a radius of 4 meters and the current and speed data from the motors has been collected and analyzed. The results of the test are shown in figure 4.

\section{Discussion}

The first and the last stretch, in which both wheels carry the same speed, correspond to the initial and final straight trajectory. It is observable that when the trajectory becomes curve, the inner wheel speed (the left wheel in this case) is lower than the outer one (right one). On the other hand, the current signal remains practically identical for both motors, which means that the torque is the same. It has been also compared the theoretical speed ratio between both wheels to the experimental value, obtaining an error of $2,03 \%$. This error is within the expected range, due to the difficulty of maintaining an exact radius of 4 meters during the entire path.

From the analysis of these data we can establish that the ED works correctly at low speed in sharp curves when there is no slip on any of the wheels.

\section{Conclusions}

It has been shown that it is possible to perform an electronic differential system without steering angle sensors nor dedicated speed sensors.

The hardware of the system is based on standard BLDC motors and controllers and the general purpose Arduino platform.

Its main characteristics are very small weight and low cost. This makes it very suitable for use in light electric vehicles with more than one drive wheel.

In order to carry out a more exhaustive evaluation of the proposed system, new tests should be carried out at a higher speeds and in conditions of slippage of drive train wheels. Our purpose is to perform these tests on a new four-wheeled vehicle that currently is under development.

\section{Acknowledgement}

This work is part of an educational innovation project financed by the Universidad de Málaga within its programs 2013-2015 (PIE13-012) and 2015-2017 (PIE15-122)

\section{References}

[1] Van den Bossche, Alex, Peter Sergeant, and Isabelle Hofman, "Towards Low Energy Mobility Using Light and Ultralight Electric Vehicles.”, First International Conference On Electromechanical Engineering, Proceedings. Ed. Abderrezak Metatla. University of Skikda Algeria, 2012.

[2] I. Hofman, P. Sergeant and A. Van den Bossche, "Drivetrain design for an ultra light electric vehicle with high efficiency," 2013 World Electric Vehicle Symposium and Exhibition (EVS27), Barcelona, 2013, pp. 1-6.

doi: 10.1109/EVS.2013.6914766

[3] Gil-Sánchez, Marina; Fernández-Ramos, José. “A specific photovoltaic panel for an ultra-light electric vehicle focused on urban mobility", The first World Light Electric Vehicle Summit (LEVS16), Barcelona, 2016 http://hdl.handle.net/10630/12435

[4] A. Muetze and Y. C. Tan, "Performance evaluation of electric bicycles," Fourtieth IAS Annual Meeting. Conference Record of the 2005 Industry Applications Conference, 2005., 2005, pp. 2865-2872 Vol. 4 doi: 10.1109/IAS.2005.1518866

[5] L. Solero, O. Honorati, F. Caricchi and F. Crescimbini, "Nonconventional three-wheel electric vehicle for urban mobility," in IEEE Transactions on Vehicular Technology, vol. 50, no. 4, pp. 1085-1091, Jul 2001.

doi: $10.1109 / 25.938582$

[6] J. Sindha, B. Chakraborty and D. Chakravarty, "Rigid body modeling of three wheel vehicle to determine the dynamic stability - A practical approach," 2015 IEEE International Transportation Electrification Conference (ITEC), Chennai, 2015, pp. 1-8.

doi: 10.1109/ITEC-India.2015.7386889 

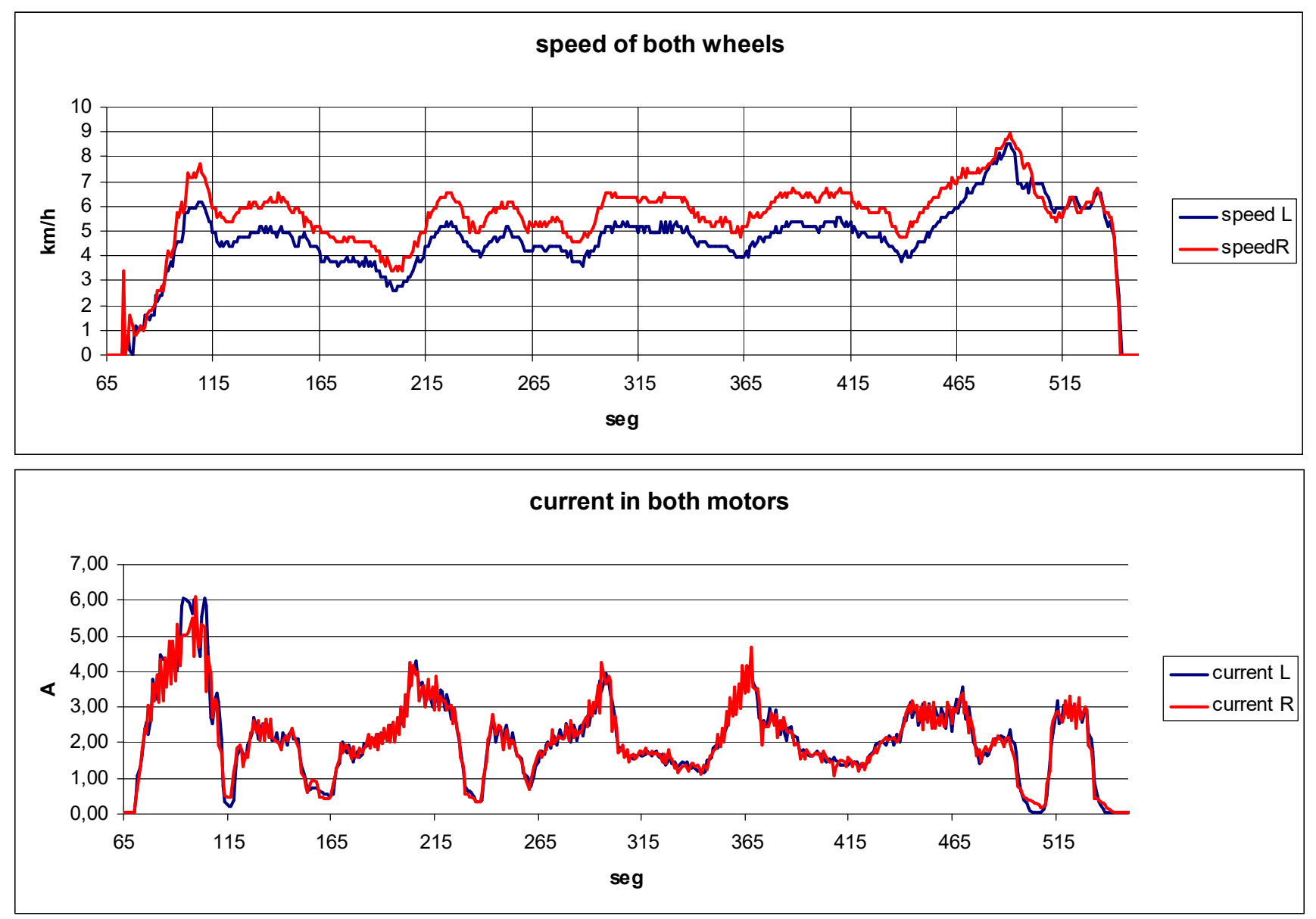

Figure 4

[7] H. Furuichi, J. Huang, T. Fukuda and T. Matsuno, "Switching Dynamic Modeling and Driving Stability Analysis of Three-Wheeled Narrow Tilting Vehicle," in IEEE/ASME Transactions on Mechatronics, vol. 19, no. 4, pp. 1309-1322, Aug. 2014.

doi: 10.1109/TMECH.2013.2280147

[8] D. Grünstäudl et al., "Design and performance analysis of a $48 \mathrm{~V}$ electric drive system for a cargo tricycle," 2017 IEEE Transportation Electrification Conference and Expo (ITEC), Chicago, IL, 2017, pp. 550-555.

doi: 10.1109/ITEC.2017.7993330

[9] F. J. Perez-Pinal, I. Cervantes and A. Emadi, "Stability of an Electric Differential for Traction Applications," in IEEE Transactions on Vehicular Technology, vol. 58, no. 7, pp. 32243233, Sept. 2009.

doi: 10.1109/TVT.2009.2013473

[10] T. I. Kosmanis and T. V. Yioultsis, "Electrical drivetrains for tad-pole and delta type recumbent tricycles," 2014 International Conference on Connected Vehicles and Expo (ICCVE), Vienna, 2014, pp. 80-85.

doi: 10.1109/ICCVE.2014.7297662

[11] C. Fu, R. Hoseinnezhad, R. Jazar, A. Bab-Hadiashar and S. Watkins, "Electronic differential design for vehicle side-slip control," 2012 International Conference on Control, Automation and Information Sciences (ICCAIS), Ho Chi Minh City, 2012, pp. 306-310.

doi: 10.1109/ICCAIS.2012.6466607

[12] J. Folgado, S. S. Valtchev and F. Coito, "Electronic differential for electric vehicle with evenly split torque," 2016 IEEE International Power Electronics and Motion Control Conference (PEMC), Varna, 2016, pp. 1204-1209.

doi: 10.1109/EPEPEMC.2016.7752167

[13] B. Tabbache, A. Kheloui and M. E. H. Benbouzid, "An Adaptive Electric Differential for Electric Vehicles Motion
Stabilization," in IEEE Transactions on Vehicular Technology, vol. 60, no. 1, pp. 104-110, Jan. 2011.

doi: 10.1109/TVT.2010.2090949

[14] A. Haddoun, M. E. H. Benbouzid, D. Diallo, R. Abdessemed, J. Ghouili and K. Srairi, "Design and implementation of an Electric Differential for traction application," 2010 IEEE Vehicle Power and Propulsion Conference, Lille, 2010, pp. 1-6.

doi: 10.1109/VPPC.2010.5729056

[15] Fernández-Ramos, José; Aghili-Khatir,Foroohar, "Electric Vehicle based on Standard Industrial Components", 2010 International conference on renewable energies and power quality (ICREPQ10), Granada, 2010 https://doi.org/10.24084/repqi08.344 